\title{
Escala das Dimensões do Desenvolvimento da Identidade: Estudos psicométricos iniciais
}

\author{
Ana Prioste ${ }^{1,2}$, Ana Lugar 3 , Paula Paulino², Inês Jongelenen ${ }^{1} \&$ Pedro J. Rosa ${ }^{1,4,5}$ \\ ${ }_{1}$ HEI-LAB - Digital Human-Environment Interaction Lab, Escola de Psicologia e Ciências da Vida, \\ Universidade Lusófona de Humanidades e Tecnologias, Lisboa, Portugal \\ ${ }^{2}$ CICPSI, Faculdade de Psicologia, Universidade de Lisboa, Lisboa, Portugal \\ ${ }^{3}$ Escola de Psicologia e Ciências da Vida, Universidade Lusófona de Humanidades e Tecnologias, Lisboa, \\ Portugal \\ ${ }^{4}$ Instituto Universitário de Lisboa (ISCTE-IUL), CIS-IUL, Lisboa, Portugal \\ ${ }^{5}$ Centro de Investigação em Psicologia (CIP), ISMAT, Portimão
}

\begin{abstract}
Resumo: Este estudo apresenta o processo de desenvolvimento e os estudos psicométricos iniciais da Escala das Dimensões do Desenvolvimento Identitário (DIDS), um instrumento que avalia os processos de desenvolvimento da identidade. Participaram no estudo 403 indivíduos (15-29 anos), que responderam a um questionário de dados sociodemográficos, à DIDS e ao Inventário da Sintomatologia Psicológica (ISP). Foram analisadas as características psicométricas da DIDS. Para o estudo da validade de construto foi realizada uma análise fatorial confirmatória. As validades convergente e discriminante foram analisadas através: da Variância Extraída Média (VEM); da comparação dos valores de VEM com os valores de correlação ao quadrado entre os fatores; e das correlações entre os processos de desenvolvimento da identidade e o ISP. A DIDS apresentou índices satisfatórios de fiabilidade compósita e consistência interna e a estrutura fatorial proposta foi confirmada. Os resultados sugerem que o instrumento poderia tornarse mais fiável com o aumento do número de itens em alguns fatores e a eliminação de alguns itens existentes. No entanto, a DIDS é um instrumento passível de ser usado na medição do desenvolvimento identitário em adolescentes e adultos emergentes portugueses.
\end{abstract}

Palavras-chave: Desenvolvimento identitário; estudo inicial de validação; adultos emergentes; adolescentes; psicopatologia.

Dimensions of Identity Development Scale: Initial psychometric studies: This paper presents the development process and the initial psychometric studies of the Dimensions of Identity Development Scale (DIDS), an instrument for assessing identity development processes. The study involved 403 participants (ages ranging from 15 to 29 years) who answered the DIDS, the Brief Symptom Inventory (BSI) and a questionnaire about socio-demographic information. We analyzed the psychometric properties of DIDS. We conducted a confirmatory factorial analysis to study the construct validity. Convergent and discriminant validity were analyzed through: the Average Variance Extracted (AVE); the comparison between the VEM values with the value of the correlation to square between the factors; and the correlations between the BSI and the identity processes. The Portuguese version of DIDS showed acceptable composite reliability, internal consistency, and the proposed factorial structure was confirmed. The results suggest that DIDS would be more reliable by increasing of the number of items in some factors and eliminating some existing items. However, DIDS is an instrument that can be used to measure identity development processes among Portuguese adolescents and emerging adults.

Keywords: Identity development; initial validation study; adolescents; emerging adults; psychopathology.

A identidade, enquanto uma tarefa desenvolvimental central na adolescência e na adultez emergente (Arnett, 2000; Erikson, 1968), tem sido definida como um conjunto de características biológicas, psicológicas e sociodemográficas que permitem definir o que a pessoa pensa sobre si e como se perceciona nas relações interpessoais que estabelece com os outros (Sedikides \& Brewer, 2001; Vignoles, Schwartz, \& Luyckx, 2011). O reconhecimento da importância da identidade no funcionamento psicossocial e interpessoal ao longo do ciclo da vida tem contribuído para o aumento do volume de trabalhos empíricos nesta área (Côte \& Levine, 2009; Luyckx, Klimstra, Duriez, Petergem, \& Beyers, 2013; Meeus, 2011; Luyckx, Schwartz, Goossens, Beyers, \& Missoten, 2011). Nas últimas décadas, têm sido

${ }^{1}$ Morada para correspondência: Ana Prioste, Campo Grande 376, 1749-024 Lisboa. E-mail: ana.prioste@ulusofona.pt 
desenvolvidos diversos modelos focados no desenvolvimento da identidade, nomeadamente, o modelo dos estatutos da identidade (Marcia, 1966), o modelo dos estilos identitários (Berzonsky et al., 2013), o modelo integrativo da identidade (Luyckx et al., 2008) e diversos instrumentos para avaliar a identidade [e.g., Ego Identity Process Questionaire (Balistreri, Busch-Rossnage, \& Geisinger, 1995); Revised Identity Style Inventory (Berzonsky et al., 2013); Dimensions of Identity Development Scale (DIDS; Luyckx et al., 2008)]. O presente estudo centra-se no processo de adaptação da DIDS ao contexto português e nos estudos psicométricos do instrumento, com uma amostra de adolescentes e de adultos emergentes (1529 anos). Pretende-se, deste modo, contribuir para o enriquecimento da literatura nesta área, através de uma compreensão mais alargada dos processos de desenvolvimento da identidade de adolescentes e adultos emergentes em Portugal.

\section{Mapas de identidade: Conceptualizações e modelos}

Diversos autores (e.g., Chen, Boucher, \& Tapias, 2006; Epp \& Price, 2008; Sedikides \& Brewer, 2001; Vignoles et al., 2011) têm convergido na conceptualização da identidade, sugerindo uma distinção entre três níveis - identidade individual, relacional e coletiva. Estes níveis variam de acordo com a interrelação e interdependência com os outros envolvidos na construção da identidade e com a identificação com o self com os outros (Vignoles et al., 2011). Por um lado, a identidade individual é focada no autoconceito e inclui valores, objetivos, crenças e padrões de comportamento e de tomada de decisão (Sedikides \& Brewer, 2001). A identidade relacional corresponde aos papéis que a pessoa desempenha com os outros e aos atributos que definem a relação (Epp \& Price, 2008). Por sua vez, a identidade coletiva corresponde à pertença a um grupo específico e à forma como o indivíduo se perceciona na relação com os outros (Brewer \& Gardner, 1996). A identidade coletiva corresponde a uma extensão social do self que depende das relações interpessoais e da pertença a um grupo (Iyer, Jetten, Tsivrikos, Postmes, \& Haslam, 2009). Contudo, a literatura (e.g., Vignoles et al., 2011) tem realçado visões distintas em relação ao facto da identidade (a) ser descoberta ou construída individual ou socialmente, (b) ser relativamente estável ou fluida e em constante mudança, e (c) dever ser estudada através de desenhos quantitativos ou qualitativos.

Erickson foi um dos pioneiros no estudo da identidade, ao desenvolver a Teoria do Desenvolvimento Psicossocial (Erickson, 1968), propondo a existência de oito estádios de desenvolvimento psicossocial associados a mudanças físicas, cognitivas e sociais e a tarefas específicas relacionadas com a definição pessoal e o relacionamento com os outros (Evans, Forney, Guido, Pertton, \& Reen, 2010). Desenvolvido a partir dos trabalhos de Erikson (1968), o modelo dos estatutos identitários (Marcia, 1980) identificou dois processos centrais na formação da identidade: compromisso (adesão a um conjunto de objetivos, valores e crenças) e exploração (questionamento ativo das alternativas identitárias). Com base nestas duas dimensões, Marcia (1980) definiu quatro estatutos: (1) Identidade realizada, caracterizado pelo estabelecimento de um compromisso com objetivos e ideias individualmente determinados, após um período de exploração de alternativas; (2) Identidade difusa, caracterizada pela ausência de exploração de alternativas e de compromissos; (3) Identidade fechada, descrita pela existência de compromissos e objetivos claros e firmes, sem um período de exploração prévia; (4) Identidade moratória, descrita por um processo de exploração e pela dificuldade de tomada de decisão e de estabelecimento de compromissos.

As críticas apontadas ao modelo de Marcia (e.g., estaticidade) e a necessidade de estudar aprofundadamente os processos de compromisso e de exploração contribuíram para o desenvolvimento de modelos dinâmicos que integrem processos identitários mais específicos (Côté \& Levine, 1988; Grotevant, 1987; Mastrotheodoros, \& Motti-Stefanidi, 2016). 0 modelo integrativo do desenvolvimento identitário (Luyckx et al., 2008) foca os processos formativos e avaliativos da identidade, distinguindo os processos e estratégias adaptativos e inadaptativos. Luyckx e colaboradores (2008) distinguiram empiricamente cinco processos de identidade - exploração em amplitude, exploração em profundidade, exploração ruminativa, compromisso e identificação com compromisso - e agruparam os quatro processos em dois ciclos consecutivos de formação da identidade. 0 primeiro ciclo, denominado por formação do compromisso, centra-se nos processos através dos quais os indivíduos exploram alternativas da identidade (exploração de amplitude ou pró-ativa) e aderem a compromissos de identidade (compromisso, i.e., adesão a valores, objetivos e crenças). 0 segundo ciclo de identidade, conceptualizado como avaliação do compromisso, foca os processos através dos quais os indivíduos reavaliam os seus compromissos de identidade (exploração em profundidade, i.e., avaliação e exploração dos compromissos actuais) e avalia o grau em que se identificam e se sentem seguros em relação aos seus compromissos (identificação com o compromisso, i.e., grau em que os compromissos se integram no seu sentido de self). 0 processo exploração ruminativa foi posteriormente adicionado ao modelo e é descrito como um bloqueador do desenvolvimento identitário ou um processo não adaptativo (Luyckx et al., 2008). Em 
indivíduos com níveis elevados de exploração ruminativa, a dificuldade em encontrar respostas satisfatórias às suas questões identitárias leva-os a um questionamento contínuo em relação às mesmas e a sentimentos de incerteza e incompetência (Luyckx et al., 2011).

A DIDS (Luyckx et al., 2008) é um instrumento de autorelato com 25 itens que foi desenvolvido para avaliar os cinco processos propostos no modelo integrativo do desenvolvimento identitário e para colmatar limitações (e.g., psicométricas, extensão das medidas) apontadas aos instrumentos que estudam o mesmo construto. Para além disso, os autores do instrumento focaram a importância da construção de um instrumento que avalie a exploração ruminativa e que sintetize e integre diversas perspetivas empíricas (Luyckx et al., 2008, 2011; Mannerström, Hautamäki, \& Leikas, 2017). 0 modelo proposto por Luyckx e colaboradores (2008) foi testado em diversos contextos culturais e os resultados dos estudos suportaram a adequação da DIDS para o estudo do construto em apreço (e.g., Mastrotheodoros \& MottiStefanidi, 2016; Mannerström et al., 2017; Morsunbul \& Cok, 2014; Pesigan, Luyckx, \& Alampay, 2014; Skhirtladze, Javakhishvili, Schwartz, Beyers, \& Luyckx, 2016; Zimmermann, Lannegrand-Willems, SafontMottay, \& Cannard, 2015).

Os estudos baseados no modelo de Luyckx e colaboradores (2008) têm mostrado uma associação entre os processos de identidade e a sintomatologia psicológica. Os processos compromisso e identificação com o compromisso têm sido negativamente associados à sintomatologia depressiva (e.g., Luyckx et al., 2008; Luyckx, Klimstra, Schwartz, \& Duriez, 2013; Mastrotheodoros, \& Motti-Stefanidi, 2016) e ansiosa (Sica, Sestito, \& Ragozini, 2014). Em relação aos processos de exploração, a exploração ruminativa tem sido associada positivamente à sintomatologia depressiva (Luyckx et al., 2011; Luyckx, Klimstra, Schwartz, \& Duriez, 2013; Mastrotheodoros, \& Motti-Stefanidi, 2016) e ansiosa (Mastrotheodoros, \& Motti-Stefanidi, 2016; Sica et al., 2014; Skhirtladze et al., 2016). Os estudos que analisam o impacto da etapa de desenvolvimento nos processos de identidade têm mostrado consistentemente que, na transição para a idade adulta, os níveis de compromisso e de identificação com o compromisso aumentam e a exploração em amplitude diminui (Luyckx et al., 2008, 2013). Estes resultados têm sido associados ao impacto da emergência de uma estrutura de identidade mais sólida (Meeus, Iedema, Helsen, \& Vollebergh, 1999), i.e. maturação da identidade (Meeus, van de Schoot, Keijsers, Schwartz, \& Branje, 2010).

0 sexo tem sido apontado como uma variável relevante no estudo dos processos de desenvolvimento identitário (Crocetti, Scrignaro, Sica, \& Magrin, 2012; Luyckx et al., 2006, 2015; Meeus et al., 2010). Os resultados do estudo de Luyckx e colaboradores (2006), com uma amostra de 5834 participantes com idades compreendidas entre os 14 e os 30 anos, mostraram que as raparigas reportam níveis mais elevados de compromisso, exploração em amplitude, exploração em profundidade e exploração ruminativa, em comparação com os rapazes. Da mesma forma, outros estudos (e.g., Meeus et al., 2010) também têm sugerido que as raparigas tendem a estabelecer compromissos mais precocemente que os rapazes. Estes dados têm sido interpretados tendo em conta as diferenças desenvolvimentais, i.e., os ritmos de maturação biológica (Luyckx et al., 2006). Neste sentido, o facto de as raparigas terem um ritmo de maturação mais acelerado em termos físicos e cognitivos (e.g., aumento da capacidade de autorreflexão) (Alsaker \& Flammer, 2006), em comparação com os rapazes, poderá contribuir para a explicação destes resultados (Luyckx et al., 2006).

\section{Adolescência e adultez emergente em Portugal}

Tendo em conta o modelo ecológico do desenvolvimento humano (Brofenbrenner, 1979), perspetivamos a inter influência de diversos níveis sistémicos no desenvolvimento da identidade. A um nível macrossistémico, consideramos o impacto dos processos sociais (e.g., valores, ideologia, sistemas político, social, económico e cultural, globalização) no desenvolvimento da identidade, enfatizando o impacto da ausência ou escassez de medidas sociais de apoio à independência dos adultos emergentes portugueses no atraso do processo de autonomização (Brandão, Saraiva, \& Matos, 2012; Saraiva \& Matos, 2016). Alguns autores (e.g., Côté \& Levine, 2002) têm apontado para que a exposição a múltiplas possibilidades de autorrealização estimulada pela orientação consumista das sociedades pós-modernas pode contribuir para o aumento de dificuldades na tomada de decisão e no estabelecimento de compromissos, repercutindo-se no aumento dos níveis de ansiedade. No mesmo sentido, Crocetti, Luyckx, Scrignaro, e Sica (2011) e Zimmermann e colaboradores (2015) referem que a incerteza em relação ao futuro e a ruminação se sustentam reciprocamente, enfraquecendo os compromissos e os níveis de ajustamento.

A ausência de expectativas e de prescrições sociais tem também contribuído para o prolongamento da condição juvenil, aumentando o período de exploração e instabilidade prévio à assunção de compromissos e lentificando o processo de transição para a idade adulta (Arnett, 2000). Em Portugal, observa-se a tendência para a existência de percursos educativos mais longos, da inserção tardia no mercado de trabalho, do prolongamento da coabitação com a família de origem e do aumento da idade 
média para o casamento e para o nascimento do primeiro filho (Brandão, Saraiva, \& Matos, 2012; Guerreiro \& Abrantes, 2004; Mendonça, Andrade, \& Fontaine, 2009; Saraiva \& Matos, 2016). Por exemplo, no ano de 2014, as mulheres portuguesas, em média, saíram de casa aos 29.8 anos e os homens aos 31.5 anos, normalmente para casar (Eurostat, 2016).

A família, enquanto parte do microssistema, ocupa uma posição central em todas as etapas do ciclo de vida, assegurando a continuidade do ser humano e construindo a ponte indivíduo-sociedade através da transmissão de crenças, da partilha emocional e da participação em rituais coletivos (Linares, 1996). A tendência atual do prolongamento da coabitação com a família de origem e a manutenção do "ninho cheio" (Carter \& McGoldrick, 1995) tem sido perspetivada de forma divergente. Kublikowski e Rodrigues (2016) sugerem que o prolongamento e rearranjo das dinâmicas familiares possam estar associados à pluralidade e à unicidade das trajetórias de desenvolvimento individual, afastando-as de um carácter disfuncional e não colocando em causa a condição adulta dos filhos. Por outro lado, o prolongamento da coabitação pode apresentar um carácter disfuncional (Vieira \& Rava, 2012), já que mantém a dependência do adulto emergente em relação à família, dificultando o compromisso social e perpetuando a condição de "adolescentes" (Jablonski \& Martino, 2013).

0 cronossistema, transversal a todos os níveis contextuais, remete-nos para a dimensão temporal. A identidade muda ao longo da vida, de acordo com as mudanças desenvolvimentais e a interação com o meio (Vignoles et al., 2011), contudo, é expectável que as grandes mudanças ocorram na adolescência e na transição para a idade adulta (Arnett, 2000; Erikson, 1968; Schwartz, Zamboanga, Luyckx, Meca, \& Ritchie, 2013). Neste sentido, Arnett (2007), considerando o impacto do contexto social atual, aponta para que, embora a experimentação de papéis sociais típicos da vida adulta seja iniciada na adolescência, os processos de exploração de alternativas sejam maioritariamente realizados após os 18 anos. Os trabalhos realizados com adultos emergentes em Portugal (e.g., Mendonça et al., 2009; Saraiva \& Matos, 2016) têm apoiado os resultados dos estudos internacionais, mostrando que, após a experienciação de um período inicial isento de responsabilidades e marcado pela aventura e experimentação, segue-se um período de acréscimo de responsabilidades e estabilidade e de implementação de projetos familiares.

Tendo em conta o interesse que o construto de identidade tem assumido nas comunidades clínica e académica, torna-se relevante a existência de uma medida robusta que o possa avaliar no contexto português. Para além disso, considerando que a identidade é desenvolvida nestes vários níveis sistémicos e atendendo às especificidades do contexto português, torna-se relevante perceber se o DIDS é um instrumento válido para avaliar os processos de desenvolvimento da identidade em adolescentes e adultos emergentes portugueses.

0 presente estudo pretende com uma amostra de adolescentes (15-17 anos) e de adultos emergentes (18-29 anos): (1) traduzir e adaptar a DIDS para a língua portuguesa; (2) analisar as propriedades psicométricas da DIDS através da análise da validade e da fiabilidade; (3) analisar as diferenças de sexo. 0 estudo da validade de construto foi realizado através da confirmação da estrutura fatorial da versão portuguesa da DIDS numa amostra de adolescentes e adultos emergentes portugueses. As validades convergente e discriminante da escala foram estudadas através da correlação entre os processos de desenvolvimento da identidade e medidas de depressão e ansiedade. Partindo do pressuposto que a depressão e ansiedade são dois construtos teoricamente paralelos aos processos de desenvolvimento da identidade, Mastrotheodoros e Motti-Stefanidi (2016) utilizaram estas variáveis para analisar as propriedades psicométricas da DIDS. No mesmo sentido, diversos autores têm realçado o facto de a sintomatologia depressiva se relacionar com diversos tipos ou estados de identidade (Kroger \& Marcia, 2011; Luyckx et al., 2008; Luyckx, Klimstra, Schwartz, \& Duriez, 2013). Tendo em conta a literatura (e.g., Luyckx et al., 2011; Luyckx, Klimstra, Schwartz, \& Duriez, 2013; Mastrotheodoros, \& MottiStefanidi, 2016; Sica, et al., 2014; Skhirtladze et al., 2016), a validade convergente da escala foi estudada através da correlação entre o processo exploração ruminativa, a depressão e a ansiedade. A validade discriminante da escala foi estudada através da correlação entre o processo de exploração em amplitude, a ansiedade e depressão, já que os resultados de estudos anteriores (e.g., Mastrotheodoros, \& MottiStefanidi, 2016; Sica et al., 2014; Skhirtladze et al., 2016) mostraram que estas variáveis não se encontram associadas.

\section{MÉTODO}

\section{Participantes}

A amostra foi constituída por 403 participantes $(N=403)$ com idades compreendidas entre os 15 e os 29 anos $(M=20.35 ; D P=3.36)$, sendo que $61 \%$ dos participantes habitam com a família nuclear intacta, $8.2 \%$ dos participantes habitam com a família monoparental e $17.6 \%$ coabitam com o/a namorado/a ou amigos/colegas. A maioria da amostra é do sexo feminino (65\%) e frequenta o ensino superior (51.6\%). 
Relativamente à situação afetivo-relacional, 54.6\% não tem relacionamento amoroso, 37\% da amostra tem uma relação de namoro e 3.5\% vive em união de facto. Em relação à zona de residência, 58.3\% da amostra vive na zona da Grande Lisboa, $16.6 \%$ vive na zona centro, $6.7 \%$ da amostra vive na Madeira, $3.2 \%$ no Alentejo e 2.5\% na zona Norte. Por último, a maioria dos participantes (61.8\%) nunca teve acompanhamento psicológico e/ou psiquiátrico, $28 \%$ já tiveram no passado e $7.2 \%$ têm acompanhamento atual.

\section{Instrumentos}

Questionário de dados sociodemográficos. Os participantes responderam a um questionário dados pessoais e sociodemográficos que integrou questões como idade, sexo, nível de escolaridade, religiosidade, zona de residência, entre outras.

Escala das Dimensões do Desenvolvimento Identitário (Dimensions of Identity Development Scale, DIDS; versão original: K. Luyckx et al., 2008). Este instrumento de autorrelato é composto por 25 itens que avaliam o desenvolvimento da identidade, numa escala de Likert de cinco pontos (de $1=$ discordo fortemente a 5 = concordo fortemente). A DIDS avalia cinco dimensões: Exploração em profundidade, composta por cinco itens (e.g., "Falo com outras pessoas sobre os meus planos para o futuro.") que avaliam a exploração de alternativas após a adesão a compromissos; Exploração em amplitude, integra cinco itens (e.g., "Estou a pensar em diferentes estilos de vida que podem ser bons para mim.") que medem a exploração de alternativas previamente à adesão a compromissos; Compromisso, inclui cinco itens (e.g., "Tenho uma imagem sobre o que vou fazer no futuro.") que avaliam a adesão a compromissos; Identificação com o compromisso, constituída por cinco itens (e.g., "Os meus planos para o futuro dão me autoconfiança.") que avaliam o grau de segurança e de identificação em relação aos compromissos; e Exploração ruminativa, composta por cinco itens (e.g., "Tenho dúvidas sobre o que quero realmente alcançar na vida.") que avaliam a exploração progressiva de diversas alternativas e a não adesão a compromissos.

No estudo de validação da DIDS (Luyckx et al., 2008), com uma amostra de jovens adultos, as dimensões da escala mostraram níveis adequados de consistência interna, variando entre $\alpha=.79$ para a dimensão Exploração em profundidade e entre $\alpha=.86$ para as dimensões Compromisso, Identificação com o compromisso e Exploração ruminativa. No mesmo estudo de validação (Luyckx et al., 2008), com uma amostra de adolescentes, as dimensões da DIDS mostraram igualmente níveis adequados de consistência interna, variando entre $\alpha=.80$ para a dimensão Exploração em profundidade e $\alpha=.86$ para as dimensões Exploração em amplitude (Luyckx et al., 2008). De relevar também que os índices de ajustamento da análise fatorial confirmatória do modelo pentafatorial foram considerados adequados para a amostra de adolescentes e para a amostra de adultos emergentes (RMSEA $=.07$ e CFI $=.94$, em ambas as amostras) (Luyckx et al., 2008).

Inventário de Sintomatologia Psicológica (ISP; Brief Symptom Inventory; Versão original: L. Derogatis, 1982; tradução e adaptação para a população portuguesa: M. C. Canavarro, 1999). O ISP é um instrumento de autorrelato composto por 53 itens. A tarefa do participante consistiu em qualificar a intensidade em que foi afetado, durante a última semana, por um conjunto de sintomas, utilizando, para tal, uma escala de Likert de cinco pontos ( $0=$ nunca a $4=$ muitíssimas vezes). Este instrumento avalia nove dimensões (Somatização, Obsessão-compulsão, Sensibilidade interpessoal, Depressão, Ansiedade, Hostilidade, Ansiedade fóbica, Ideação paranoide e Psicoticismo) e três índices globais (Índice geral de sintomas, Índice de sintomas positivos e Índice total de sintomas positivos), que constituem avaliações sumárias de perturbação emocional (Canavarro, 1999). Neste estudo, foram apenas utilizadas as dimensões Depressão e Ansiedade.

No estudo de validação de Canavarro (1999), com uma amostra de 551 indivíduos, o ISP revelou níveis de consistência interna adequados entre $\alpha=.62$ para a dimensão Psicoticismo e $\alpha=.79$ para a dimensão Somatização. No presente estudo, as dimensões Depressão e Ansiedade apresentaram níveis de consistência interna adequados $\left(\alpha=.70, r_{m . i}=.35\right.$; e $\alpha=.71, r_{m . i}=.28$, respetivamente $)$.

\section{Procedimento}

A tradução da DIDS foi realizada, individualmente, por duas psicólogas que dominam a língua inglesa e o modelo teórico de Luyckx e colaboradores (2008). Após a tradução, e tendo em conta a equivalência lexical, conceptual e gramatical, procurou encontrar-se um consenso de modo a que os itens da versão portuguesa abarcassem o sentido dos itens da escala original. Posteriormente, uma especialista em língua inglesa realizou a retroversão dos itens. Após estes procedimentos, a escala foi aplicada a um grupopiloto de 20 participantes composto por adolescentes e adultos emergentes, com o qual se discutiu a 
adequação vocabular e a compreensão dos itens. Na sequência desta discussão, foram alterados alguns itens. A escala foi também analisada por duas especialistas na área da Psicologia Clínica. A recolha de dados decorreu após a aprovação do projeto de investigação pela Comissão de Ética e Deontologia em Investigação Clínica da Escola de Psicologia e de Ciências da Vida da Universidade Lusófona de Humanidades e Tecnologias. A amostra foi selecionada a partir de uma amostra mais alargada de 454 participantes, tendo sido estabelecidos os seguintes critérios de inclusão no presente estudo: (a) ter nacionalidade portuguesa; e (b) ter idades compreendidas entre os 15 e os 30 anos. Uma amostra de 403 participantes cumpriu estes critérios, pelo que foi integrada no presente estudo.

A amostra foi recolhida durante 15 meses (de fevereiro de 2016 a abril de 2017), através de uma técnica de amostragem não probabilística, denominada de "bola de neve". A amostra foi recolhida através de diferentes procedimentos: $74.94 \%$ da amostra foi recolhida presencialmente, através de procedimentos informais em grupo (e.g., contexto de sala de aula em Instituições de Ensino Superior) e individualmente (e.g., amigos e conhecidos); e a restante foi recolhida on-line através da plataforma Google Docs, após a divulgação do estudo através de redes sociais e de correio eletrónico.

$\mathrm{Na}$ recolha da amostra presencial, as investigadoras mostraram disponibilidade para esclarecer as dúvidas relacionadas com as questões e/ou vocabulário. $\mathrm{Na}$ recolha on-line, foram disponibilizados os contactos da investigadora responsável pelo estudo, caso surgisse alguma dúvida ou questão no decorrer da participação do estudo. Após a explicitação dos objetivos do estudo, da garantia da confidencialidade e da possibilidade de desistência a qualquer momento e da assinatura do consentimento informado, os/as participantes colaboraram voluntariamente e sem remuneração.

\section{Procedimento de análise de dados}

Inicialmente, realizou-se a estatística descritiva dos 25 itens da versão portuguesa da DIDS através do cálculo da média, desvio-padrão, máximo, mínimo, curtose, erro padrão da curtose, assimetria e erro padrão da assimetria. A validade de construto foi avaliada através de uma análise fatorial confirmatória e da validade convergente e discriminante. De acordo com Fornell e Larcker (1981), a validade de construto é defensável se apresentar, em primeiro lugar, validade fatorial, depois validade convergente e validade discriminante com outros fatores presentes na DIDS.

A validade fatorial foi avaliada por intermédio de uma Análise Fatorial Confirmatória (AFC) através do método da Máxima Verossimilhança (ML), com recurso ao software estatístico AMOS 23.0. Para analisar o ajustamento do modelo proposto e compará-lo com os modelos alternativos, utilizaram-se os seguintes indicadores: o comparative fit índex (CFI); a razão do qui-quadrado pelos graus de liberdade $\left(\chi^{2} / g l\right)$, o goodness of fit índex (GFI), root mean square residual (RMR) e o root meam square error of approximation (RMSEA). De acordo com Garson (2012), valores de CFI iguais ou superiores a .90, valores de $\chi^{2} / g l$ menores que cinco e valores de RMSEA iguais ou inferiores a 0.06 revelam um bom ajustamento do modelo. Tendo em conta Marôco (2010), quanto menor for o RMR, melhor será o ajustamento do modelo. Para além destes indicadores, o expected cross-validation índex (ECVI) foi utilizado para avaliar diferenças entre os modelos concorrentes testados. Garson (2012) sugere que valores mais baixos de ECVI indicam um bom ajustamento do modelo. 0 índice de modificação (MI) foi utilizado para inclusão de parâmetros adicionais no modelo.

A validade convergente foi avaliada através da Variância Extraída Média (VEM) dos itens por cada um dos fatores (Marôco, 2010). De acordo com as orientações de Fornell e Larcker (1981), VEM $\geq .50$ é indicador de validade convergente. A VEM foi calculada manualmente seguindo as orientações de Hair, Black, Babin e Anderson (2009). Já a validade discriminante foi avaliada comparando a VEM de cada fator com o quadrado da correlação de Pearson $\left(R^{2}\right)$ entre fatores. Assume-se que existe validade discriminante quando a VEM de cada fator é superior ao $R^{2}$ entre fatores (Fornell \& Larcker, 1981). Para avaliar a validade convergente e discriminante das dimensões da DIDS foram calculados, igualmente, os coeficientes de correlação de Pearson entre as dimensões da escala e as dimensões Depressão e Ansiedade do ISP.

No que concerne à fiabilidade, esta foi estimada através da Fiabilidade Compósita (FC) e do alfa de Cronbach $(\alpha)$ (Hair et al., 2009). Considerou-se uma fiabilidade adequada quando FC e $\alpha \geq$.70. A FC foi computada manualmente seguindo as recomendações de Marôco (2010). Para analisar a consistência interna das cinco dimensões da escala foi calculado o alfa de Cronbach $(\alpha)$ e as correlações médias interitem, $r_{m . i .} 0$ estudo das diferenças de sexo foi realizado através do teste $t$-Student para amostras independentes. 0 cálculo do alfa do Cronbach e o teste às diferenças de sexo foram realizados com recurso ao software Statistical Package for the Social Sciences (SPSS), versão 22. Em todos os procedimentos estatísticos, foi estabelecido um nível de significância de $5 \%(p<.05)$. 


\section{RESULTADOS}

A Tabela 1 apresenta a estatística descritiva (média, desvio-padrão, assimetria, erro padrão da assimetria, curtose, erro padrão da curtose, mínimo e máximo) dos itens da versão portuguesa da DIDS.

Tabela 1. Estatística Descritiva dos Itens da Versão Portuguesa da DIDS

\begin{tabular}{|c|c|c|c|c|}
\hline Item & $M(D P)$ & Min.-Max. & Assimetria (Erro) & Curtose (Erro) \\
\hline Item 1 & $3.76(1.22)$ & $1-5$ & $-.49(.12)$ & $.29(.24)$ \\
\hline Item 2 & $3.91(.84)$ & $1-5$ & $-.65(.12)$ & $.53(.24)$ \\
\hline Item 3 & $3.57(.90)$ & $1-5$ & $-.34(.12)$ & $-.15(.24)$ \\
\hline Item 4 & $3.84(.79)$ & $1-5$ & $-.59(.12)$ & $.64(.24)$ \\
\hline Item 5 & $3.85(.92)$ & $1-5$ & $-.59(.12)$ & $-.12(.24)$ \\
\hline Item 6 & $4.02(.80)$ & $1-5$ & $-.69(.12)$ & $.62(.24)$ \\
\hline Item 7 & $4.13(.77)$ & $1-5$ & $-.85(.12)$ & $1.27(.24)$ \\
\hline Item 8 & $3.63(.97)$ & $1-5$ & $-.53(.12)$ & $-.07(.24)$ \\
\hline Item 9 & $3.97(.82)$ & $1-5$ & $-1.11(.12)$ & $2.02(.24)$ \\
\hline Item 10 & $3.71(.95)$ & $1-5$ & $-.71(.12)$ & $.29(.24)$ \\
\hline Item 11 & $2.84(1.25)$ & $1-5$ & $.13(.12)$ & $-1.13(.24)$ \\
\hline Item 12 & $3.91(1.03)$ & $1-5$ & $-.95(.12)$ & $.39(.24)$ \\
\hline Item 13 & 3.27 (1.17) & $1-5$ & $-.20(.12)$ & $-.78(.24)$ \\
\hline Item 14 & $3.28(1.10)$ & $1-5$ & $-.35(.12)$ & $-.61(.24)$ \\
\hline Item 15 & $2.77(1.14)$ & $1-5$ & $.26(.12)$ & $-.73(.24)$ \\
\hline Item 16 & $3.89(.96)$ & $1-5$ & $-.89(.12)$ & $.76(.24)$ \\
\hline Item 17 & $3.72(.93)$ & $1-5$ & $-.53(.12)$ & $.14(.24)$ \\
\hline Item 18 & $3.44(.93)$ & $1-5$ & $-.21(.12)$ & $.03(.24)$ \\
\hline Item 19 & $3.71(.90)$ & $1-5$ & $-.43(.12)$ & $.13(.24)$ \\
\hline Item 20 & $3.69(.91)$ & $1-5$ & $-.48(.12)$ & $.17(.24)$ \\
\hline Item 21 & $3.71(.84)$ & $1-5$ & $-.67(.12)$ & $.70(.24)$ \\
\hline Item 22 & 3.65 (.99) & $1-5$ & $-.79(.12)$ & $.43(.24)$ \\
\hline Item 23 & $3.52(1.00)$ & $1-5$ & $-.74(.12)$ & $.29(.24)$ \\
\hline Item 24 & $3.00(1.11)$ & $1-5$ & $-.16(.12)$ & $-.64(.24)$ \\
\hline Item 25 & $3.71(.98)$ & $1-5$ & $-.84(.12)$ & $.73(.24)$ \\
\hline
\end{tabular}

Em relação à assimetria, tal como se pode observar na Tabela 1, os itens apresentam uma distribuição assimétrica à esquerda, com exceção dos itens 11 e 15 que apresentam uma distribuição com enviesamento positivo (Marôco, 2007). De realçar também que o item 9 não apresenta uma distribuição normal (Marôco, 2007).

No que concerne ao achatamento, a maioria dos itens tem uma distribuição leptocúrtica, com exceção dos itens 3, 5, 8, 11, 13, 14, 15 e 24 que apresentam uma distribuição mesocúrtica (Marôco, 2007). Para além disso, os itens 7, 9 e 11 não apresentam uma distribuição normal (Marôco, 2007).

\section{Validade de construto}

A validade de construto foi analisada através da confirmação da estrutura fatorial da versão portuguesa da DIDS numa amostra alargada de adolescentes e adultos emergentes portugueses. Para testar o modelo com os cinco fatores proposto por Luyckx e colaboradores (2008), foi conduzida uma análise fatorial confirmatória. O modelo proposto, denominado de Modelo 1, constituído por cinco fatores correlacionados, foi comparado com três modelos alternativos: Modelo 2, que integra cinco fatores não correlacionados; Modelo 3, com uma estrutura unifatorial de primeira ordem; e Modelo 4, composto por cinco fatores de primeira ordem e um fator de segunda ordem. Os índices de ajustamento dos quatros modelos testados encontram-se apresentados na Tabela 2.

Em comparação com os Modelos 2, 3 e 4, o Modelo 1 apresenta os melhores indicadores de ajustamento, pelo que se optou por manter o modelo proposto. No entanto, ao analisar o Modelo 1, a observação dos índices de modificação (MIs) para as saturações (Lambdas, $\lambda$ ) e os erros de medida (Delta, $\delta$ ) indicou que seria aconselhável correlacionar os erros de medida dos seguintes itens: item 9 ("Penso em 
diferentes objetivos que posso procurar alcançar.") e item 10 ("Estou a pensar em diferentes estilos de vida que podem ser bons para mim."); item 16 ("Os meus planos para o futuro coincidem com os meus verdadeiros interesses e valores.") e item 17 ("Os meus planos para o futuro dão-me autoconfiança."); item 18 ("Graças aos meus planos para o futuro, sinto-me seguro/a acerca de mim próprio/a.") e item 19 ("Sinto que a direção que quero tomar na minha vida será mesmo adequada a mim."); item 21 ("Penso nos planos para o futuro que já fiz.") e item 23 ("Penso se os planos que tenho para a minha vida são realmente adequados para mim."); e item 22 ("Falo com outras pessoas sobre os meus planos para o futuro.") e item 24 ("Procuro saber o que as outras pessoas pensam sobre a direção específica que decidi tomar na minha vida."). De acordo com os MIs, adicionaram-se cinco parâmetros ao modelo, correlacionando os resíduos dos itens (item 8-item 9; item 16-item 17; item 18- item19; item 21-item 23; item 22-item 24), devido à sua proximidade frásica (Marôco, 2010). Procedendo desta forma, a estrutura fatorial resultante permitiu obter melhores indicadores de ajustamento aos dados para o modelo proposto: $\chi^{2}(259)=735.10, p<.001, \chi^{2} / g l=2.84, \mathrm{CFI}=.90, \mathrm{GFI}=.87, \mathrm{RMR}=.09, \mathrm{RMSEA}=.06 \mathrm{e} \mathrm{ECVI}=$ 2.16. Na Figura 1 é apresentada a estrutura fatorial deste modelo.

Tabela 2. Índices de Ajustamento dos Modelos Testados

\begin{tabular}{lccccccc}
\hline Modelos & $\boldsymbol{\chi 2}(\boldsymbol{g l})$ & $\chi \mathbf{2} / \boldsymbol{g l}$ & CFI & GFI & RMR & $\begin{array}{c}\text { RMSEA } \\
\text { (IC-90\%) }\end{array}$ & ECVI \\
\hline Modelo 1 & $971.34(265)$ & 3.67 & .84 & .83 & .10 & .08 & 2.72 \\
Modelo 2 & $1515.35(275)$ & 5.51 & .72 & .74 & .19 & .11 & 4.14 \\
Modelo 3 & $2121.69(274)$ & 7.74 & .58 & .63 & .14 & .14 & 6.61 \\
Modelo 4 & $2121.69(274)$ & 7.74 & .58 & .63 & .14 & .13 & 5.53 \\
\hline
\end{tabular}

Nota. Modelo 1 = cinco fatores correlacionados; Modelo 2 = cinco fatores não correlacionados; Modelo 3 = estrutura unifatorial de primeira ordem; Modelo 4 = cinco fatores de primeira ordem a predizer um fator de segunda ordem.

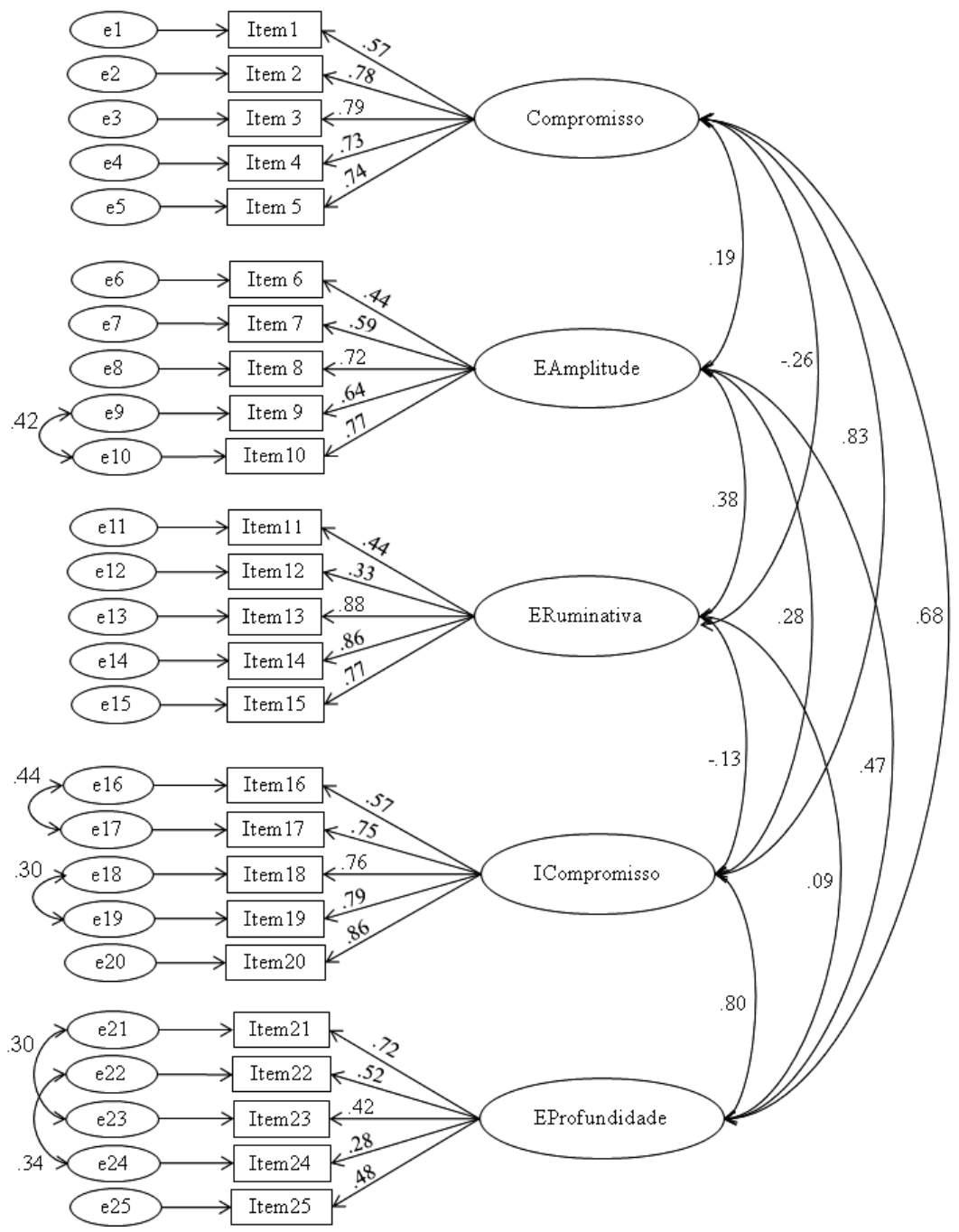

Figura 1. Estrutura fatorial do modelo com cinco fatores correlacionados e as suas saturações. 
Na Figura 1, podem também observar-se os pesos fatoriais padronizados $(\lambda)$ e as correlações $(\varphi)$ entre os vários fatores. Os fatores Compromisso e Identificação com o compromisso (ICompromisso) mostraram pesos fatoriais padronizados adequados (todos os $\lambda>.4$ ), apresentando uma média de $.72 \mathrm{e}$ de .74, respetivamente; os fatores Exploração em amplitude (EAmplitude) e Exploração ruminativa (ERuminativa) revelaram pesos fatoriais padronizados com uma média de .63 e de .65, respetivamente, indicando adequabilidade; o Exploração em profundidade (EProfundidade) apresentou pesos fatoriais padronizados mais baixos, com uma média de .48 .

Validade convergente e discriminante

A Tabela 3 apresenta a VEM, a FC e o $R^{2}$ entre os fatores.

Tabela 3. Variância Extraída Média e Fiabilidade Compósita de Cada Fator e $R^{2}$ entre Fatores

\begin{tabular}{lccccccc}
\hline \multirow{2}{*}{ Fator } & VEM & FC & \multicolumn{5}{c}{$\boldsymbol{R}^{\mathbf{2}}$} \\
\cline { 5 - 8 } & & & $\mathbf{1}$ & $\mathbf{2}$ & $\mathbf{3}$ & $\mathbf{4}$ & $\mathbf{5}$ \\
\hline 1. Compromisso & 0.65 & 0.87 & - & & & \\
2. EAmplitude & 0.53 & 0.75 & 0.03 & - & & \\
3. ERuminativa & 0.58 & 0.78 & 0.06 & 0.14 & - & & \\
4. ICompromisso & 0.67 & 0.88 & 0.69 & 0.07 & 0.02 & - & - \\
5. EProfundidade & 0.33 & 0.46 & 0.46 & 0.22 & 0.01 & 0.64 & - \\
\hline
\end{tabular}

Todos os fatores, excetuando a Exploração em profundidade, apresentaram uma VEM $\geq .5$ e FC $\geq .7$, indicando adequada validade convergente. Já o fator Exploração em profundidade, apresentou uma VEM $=.33$ e uma $\mathrm{FC}=.46$, aquém dos valores esperados para garantir validade convergente (Fornell \& Larcker, 1981).

Relativamente à validade discriminante, o Identificação com o compromisso e a Exploração em profundidade não apresentaram uma VEM superior ao $\mathrm{R}^{2}$ entre outros fatores, não demonstrando, assim, validade discriminante. Os coeficientes de correlação de Pearson entre as dimensões da escala e as dimensões Depressão e Ansiedade avaliadas pelo ISP (ver Tabela 4) permitiram complementar os resultados anteriores. Especificamente, a validade convergente foi examinada através da correlação entre a Exploração ruminativa, a Depressão e a Ansiedade, e a validade discriminante foi estudada através da correlação entre a Exploração em amplitude, a Ansiedade e Depressão, tendo em conta a literatura (e.g., Luyckx et al., 2011; Luyckx, Klimstra, Schwartz, \& Duriez, 2013; Mastrotheodoros, \& Motti-Stefanidi, 2016; Sica et al., 2014; Skhirtladze et al., 2016).

Os resultados revelaram que as dimensões Compromisso e Identificação com o compromisso se encontram significativa e negativamente associadas às dimensões Ansiedade e Depressão. Verifica-se que a dimensão Exploração ruminativa se encontra significativa e positivamente associada às dimensões Ansiedade e Depressão. Observa-se também uma associação positiva entre a Exploração em profundidade e a dimensão Ansiedade. Por último, não se encontraram associações significativas entre a dimensão Exploração em amplitude e as dimensões Ansiedade e Depressão.

Tabela 4. Correlação entre as Variáveis Índice Geral de Sintomas (IGS), Ansiedade, Depressão, Compromisso, Exploração em Amplitude (EAmplitude), Exploração Ruminativa (ERuminativa), Identificação com o Compromisso (ICompromisso) e Exploração em Profundidade (EProfundidade) $(\mathrm{N}=$ 403).

\begin{tabular}{|c|c|c|c|c|c|c|c|}
\hline Variável & 1 & 2 & 3 & 4 & 5 & 6 & 7 \\
\hline 1. Ansiedade & - & & & & & & \\
\hline 2. Depressão & $.73^{* *}$ & - & & & & & \\
\hline 3. Compromisso & $-.14^{*}$ & $-.25^{* *}$ & - & & & & \\
\hline 4. EAmplitude & .03 & -.03 & $.21^{* *}$ & - & & & \\
\hline 5. ERuminativa & $.24^{* *}$ & $.31^{* *}$ & $-.31^{* *}$ & $.30^{* *}$ & - & & \\
\hline 6. ICompromisso & $-.16^{*}$ & $-.29 * *$ & $.70^{* *}$ & $.28^{* *}$ & $-.21^{* *}$ & - & \\
\hline 7. EProfundidade & $.12 *$ & -.03 & $.35^{* *}$ & $.37^{* *}$ & $.15^{* *}$ & $.46^{* *}$ & - \\
\hline
\end{tabular}

Nota. ${ }^{*} p<.05 ; * * p<.01$ 


\section{Fiabilidade}

0 alfa de Cronbach $(\alpha)$ e homogeneidade (correlação média interitem, $r_{m i}$ ), calculados para cada fator, mostraram uma consistência interna adequada para as dimensões da escala: Compromisso $\left(\alpha=.84, r_{m i}=\right.$ .54), Exploração em amplitude $\left(\alpha=.79, r_{m i}=.43\right)$; Exploração ruminativa $\left(\alpha=.74, r_{m i}=.36\right)$, Identificação com o Compromisso $\left(\alpha=.87, r_{m i}=.56\right)$. No entanto, o fator Exploração em profundidade apresentou um valor minimamente aceitável $\left(\alpha=.68, r_{m i}=.30\right)$, sendo consistente com os resultados entrados para a FC $(<.07)$.

\section{Análise das diferenças de sexo}

Na Tabela 5 encontram-se as estatísticas descritivas (médias e desvios-padrão) em relação às cinco dimensões da escala para os sexos feminino e masculino e para o total da amostra. No estudo das diferenças de sexo foram comparadas as médias da pontuação de cada dimensão, em função do sexo, através do teste $t$-Student para amostras independentes. Os resultados demonstram que existem diferenças estatisticamente significativas na dimensão Exploração ruminativa entre sexos. 0 sexo masculino apresenta níveis de Exploração ruminativa mais elevados $(M=3.41, D P=.78)$ que o feminino $(M=3.11, D P=.80), t(393)=3.45, p<.05, d=.38$.

Tabela 5. Médias e Desvios-padrão das Dimensões da Escala para a Amostra Total e os Sexos Feminino e Masculino.

\begin{tabular}{|c|c|c|c|c|c|c|c|c|c|}
\hline & \multicolumn{2}{|c|}{ Feminino $(n=262)$} & \multicolumn{2}{|c|}{ Masculino ( $n=133$ ) } & \multicolumn{2}{|c|}{ Total $(n=285)$} & \multirow{2}{*}{$t$} & \multirow{2}{*}{$p$} & \multirow{2}{*}{$\begin{array}{c}d \\
\text { Cohen }\end{array}$} \\
\hline & $M$ & $D P$ & $M$ & $D P$ & $M$ & $D P$ & & & \\
\hline Compromisso & 3.84 & .74 & 3.71 & .73 & 3.79 & .74 & -1.61 & .11 & .18 \\
\hline Eamplitude & 3.88 & .64 & 3.93 & .63 & 3.89 & .63 & .62 & .53 & .08 \\
\hline Eruminativa & 3.11 & .80 & 3.41 & .78 & 3.22 & .79 & 3.45 & .001 & .38 \\
\hline ICompromisso & 3.68 & .75 & 3.70 & .73 & 3.69 & .74 & .30 & .76 & .03 \\
\hline Eprofundidade & 3.54 & .62 & 3.48 & .72 & 3.52 & .65 & -.91 & .36 & .09 \\
\hline
\end{tabular}

\section{DISCUSSÃo}

A DIDS é um instrumento de autorrelato que avalia os processos de desenvolvimento da identidade. Este trabalho pretendeu traduzir e adaptar para a língua portuguesa a DIDS e estudar as propriedades psicométricas do instrumento. Mais especificamente, foi analisada a validade de construto, as validades convergente e discriminante, assim como a fiabilidade da medida, numa amostra de adolescentes e adultos emergentes portugueses. Para além disso, foram analisadas as diferenças de sexo dos processos de desenvolvimento da identidade. Este estudo teve como finalidade contribuir para o enriquecimento da literatura na área da identidade na adolescência e na transição para a idade adulta em Portugal. Para além disso, procurou colmatar algumas das lacunas encontradas na literatura (e.g., inexistência de instrumentos adaptados para a população portuguesa que avaliem os processos identitários).

Em relação à análise da validade de construto, a AFC da DIDS revelou, para um modelo de primeira ordem com cinco fatores correlacionados, um bom ajustamento à estrutura da variância-covariância observada entre os 25 itens. Todos os itens apresentaram pesos fatoriais padronizados adequados, excetuando o item 24 no fator Exploração em profundidade. Embora este modelo de primeira ordem tenha apresentado médias de pesos fatoriais padronizados satisfatórias e melhores indicadores de ajustamento, o fator Exploração em profundidade apresentou limitações em termos de validade convergente, dado que o comportamento dos itens não foi explicado essencialmente pelo fator (VEM <.5), devido a pesos fatoriais padronizados mais baixos. Além disso, as correlações observadas entre os erros dos itens pertencentes aos mesmos fatores podem estar relacionadas com a similitude na formulação ou no conteúdo dos itens (Marôco, 2010).

No entanto, o fator Exploração em profundidade mostrou estar significativamente associado à dimensão ansiedade, o que corrobora trabalhos recentes (e.g., Mannerström et al., 2017). De facto, originalmente, Luyckx e colaboradores (2006) consideraram que a avaliação reflexiva em relação aos compromissos atuais se repercutia no aumento dos níveis de bem-estar; contudo, tal como defendem Mannerström e colaboradores (2017) a exploração em profundidade pode implicar a reconsideração dos compromissos, o que se pode refletir no aumento dos níveis de ansiedade. Uma possível solução para melhorar a validade convergente do fator Exploração em profundidade poderia passar pela remoção dos itens 23 e 24 ou a introdução de novos itens, no sentido de elevar a VEM e a FC para valores próximos dos desejáveis. 
Já na correlação com as dimensões do ISP, observou-se uma associação positiva, significativa e moderada entre a Exploração ruminativa e as dimensões Ansiedade e Depressão, o que corrobora os resultados de estudos anteriores (Luyckx et al., 2008, 2011; Luyckx, Klimstra, Schwartz, \& Duriez, 2013; Mastrotheodoros, \& Motti-Stefanidi, 2016; Sica et al., 2014; Skhirtladze et al., 2016). As associações encontradas apoiam a ideia circular de que, por um lado, níveis elevados de exploração ruminativa podem contribuir para o bloqueio do desenvolvimento identitário (Luyckx et al., 2008) e para o desenvolvimento de sintomatologia psicológica através da perceção de incerteza e incompetência (Luyckx et al., 2011). Por outro, níveis elevados de sintomatologia ansiosa e depressiva podem contribuir para o aumento da preocupação e do medo em relação a escolhas e compromissos (Luyckx et al., 2008), bloqueando o desenvolvimento da identidade.

No que concerne à validade discriminante, esta não se verificou para o Compromisso, Identificação com o compromisso e Exploração em amplitude. Este facto é justificável pelas magnitudes elevadas das correlações entre Compromisso - Identificação com o compromisso $(r>.83)$ e Compromisso Exploração em amplitude $(r=.80)$, o que reflete que os fatores, definidos por este conjunto de itens, não são distintos. Estes resultados poderiam indicar a existência de um fator de segunda ordem, contudo, o modelo de segunda ordem, com cinco fatores associados, mostrou-se o modelo mais ajustado aos dados. $\mathrm{Na}$ associação com as dimensões do ISP, não foram encontradas associações significativas entre o processo Exploração em amplitude, a Ansiedade e a Depressão, sugerindo validade discriminante (Mastrotheodoros, \& Motti-Stefanidi, 2016; Sica et al., 2014).

Os resultados mostraram igualmente que os processos Compromisso e Identificação com o compromisso estão associados negativamente à sintomatologia depressiva, corroborando estudos anteriores (e.g., Luyckx et al., 2008, 2011; Luyckx, Klimstra, Schwartz, \& Duriez, 2013; Mastrotheodoros, \& Motti-Stefanidi, 2016). Estes resultados poderão apontar para que, nestas etapas desenvolvimentais, as trajetórias adaptativas de desenvolvimento da identidade se caracterizem por níveis mais elevados de compromisso e de identificação com o compromisso. Considerando os sintomas associados aos quadros depressivos (e.g., tristeza, baixo autoestima, alterações somáticas e cognitivas que afetam de forma significativa a capacidade de funcionamento da pessoa) e ansiosos (e.g., comportamentos de evitamento por antecipação de uma ameaça), é expectável que a presença sintomatologia ansiosa e depressiva comprometa a capacidade de exploração e de tomada de decisão (Santos, Ferreira, \& Gonçalves, 2014).

Os resultados obtidos apontam para que a dimensão Exploração em Profundidade e a dimensão Ansiedade se associem positivamente, sendo que este resultado não é apoiado pelos estudos anteriores (Luyckx et al., 2008, 2011; Sica et al., 2014). Tendo em conta a conceptualização desenvolvida por Luyckx e colaboradores (2008), a Exploração em Profundidade remete para a recolha de informações sobre os objetivos e planos pessoais e a exposição dessa informação a outros significativos. Colocamos como hipótese explicativa o facto de os processos de exploração ativa, de reflexão e de partilha com outros poderem despoletar alguma sintomatologia ansiosa por exposição e/ou aceitação social.

No que respeita à fiabilidade, no geral, a versão portuguesa da DIDS mostrou possuir bons valores tanto na consistência interna como na fiabilidade compósita ( $>0.7$ ). Contudo, à semelhança de outros estudos (e.g., Mannerström et al., 2017), a dimensão Exploração em profundidade apresentou um valor de alfa mais baixo. Estes resultados sustentam a ideia de que, embora a estrutura pentafatorial da DIDS apresente um bom ajustamento, a consistência podia ser incrementada com mais itens (e melhores), o que asseguraria uma maior precisão do instrumento.

O facto de o sexo masculino apresentar níveis mais elevados de Exploração ruminativa não vai ao encontro do trabalho de Luyckx e colaboradores (2006). Este resultado poderá ser explicado atendendo às diferenças de sexo nos ritmos de maturação biológica (Alsaker \& Flammer, 2006) e ao seu impacto no desenvolvimento da identidade (Klimstra et al. 2010; Meeus et al., 2010). Neste sentido, hipotetizamos que o facto do sexo feminino ter um ritmo de maturação mais acelerado em termos do desenvolvimento cognitivo possa contribuir para o desenvolvimento de uma identidade mais madura, o que se reflete num nível mais baixo de exploração ruminativa. Esta questão merece ser integrada como uma questão de investigação num estudo futuro.

A DIDS pode constituir-se um instrumento de avaliação das dimensões do desenvolvimento da identidade, permitindo caracterizar e diferenciar pessoas e grupos em contextos clínicos e de investigação. Sendo este estudo um contributo inicial para o estudo das propriedades psicométricas da versão portuguesa da DIDS, apresenta limitações que restringem a sua validade: a amostra foi selecionada através de uma técnica de amostragem não probabilística, denominada de "bola de neve" e, pelo facto de não ser representativa, os resultados têm um carácter marcadamente exploratório. Para além disso, a amostra é maioritariamente constituída por participantes do sexo feminino, o que constrange a validade deste trabalho. Outras limitações prendem-se com o facto de não terem sido estudadas a estabilidade temporal do instrumento (teste-reteste) e de só ter sido utilizado um 
instrumento para testar a validade. Em relação à validade convergente, o facto de esta não ter sido avaliada através da correlação entre a DIDS e outro instrumento que meça um construto semelhante também constitui uma limitação deste estudo.

Os estudos futuros com DIDS deverão ser desenvolvidos de forma a superar estas limitações. Será necessário também replicar este estudo com amostras mais equitativas em termos da distribuição por sexo e idade. Tendo em conta a diferença entre sexos encontrada e a hipótese explicativa proposta, seria interessante desenvolver um estudo focado no impacto dos ritmos de maturação nos processos desenvolvimento da identidade na adolescência. Os trabalhos futuros também deverão contribuir para o processo de validação da DIDS, analisando a validade convergente deste instrumento através da correlação com outro que avalie um construto similar, por exemplo, o Revised Identity Style Inventory (Berzonsky et al., 2013), o Utrecht-Management of Identity Commitments Scale (Crocetti, Schwartz, Fermani, \& Meeus, 2010) e o Ego Identity Process Questionnaire (Balistreri, Busch-Rossnagel, \& Geisinger, 1995).

Em conclusão, apesar das limitações apontadas, a DIDS é um instrumento que produz dados fiáveis e válidos, podendo ser utilizado por clínicos e por investigadores, em amostras de adolescentes e adultos emergentes.

\section{REFERÊNCIAS}

Alsaker, F. D., \& Flammer, A. (2006). Pubertal maturation. In S. Jackson \& L. Goossens (Eds.), Handbook of adolescent development (pp. 30-50). Hove and New York: Psychology Press.

Arnett, J. J. (2000). Emerging adulthood: A theory of development from the late teens through the twenties. American Psychologist, 55, 469-480. http://dx.doi.org/10.1037//0003-066X.55.5.469

Arnett, J. J. (2005). The developmental context of substance use in emerging adulthood. Journal of Drug Issues, 22, 235-254. http://dx.doi.org/10.1177/002204260503500202

Arnett, J. J. (2007). Emerging adulthood: What is it, and what is it good for? Child Development Perspectives, 2, 68-73. http://dx.doi.org/10.1111/j.1750-8606.2007.00016.x

Balistreri, E., Busch-Rossnagel, N. A., \& Geisinger, K. F. (1995). Development and preliminary validation of the Ego Identity Process Questionnaire. Journal of Adolescence, 18(2), 179-192. http://dx.doi.org/10.1006/jado.1995.1012

Brandão, T., Saraiva, L., \& Matos, M. P. (2012). O prolongamento da transição para a idade adulta e o conceito de adultez emergente: Especificidades do contexto português e brasileiro. Análise Psicológica, 3, 301-313.

Berzonsky, M. D., Soenens, B., Luyckx, K., Smits, I., Papini, D. R., \& Goossens, L. (2013). Development and validation of the revised Identity Style Inventory (ISI-5): Factor structure, reliability, and validity. Psychological Assessment, 25(3), 893-904. http://dx.doi.org/10.1037/a0032642

Brewer, M. B., \& Gardner, W. (1996). Who is this "we"? Level of collective identity and self representations. Journal of Personality and Social Psychology, 71(1), 83-93. http://dx.doi.org/10.1037/0022-3514.71.1.83

Canavarro, M. C. (2007). Inventário de Sintomas Psicopatológicos: Uma revisão crítica dos estudos realizados em Portugal. In M. Simões, C. Machado, M. Gonçalves, \& L. Almeida (Eds.), Avaliação psicológica: Instrumentos validados para a população Portuguesa (vol. III, pp. 305-331). Coimbra: Quarteto Editora.

Carter, E., \& McGoldrick, M. (1995). The changing family life cycle - A framework for family therapy. Boston: Allyn and Bacon.

Chen, S., Boucher, H. C., \& Tapias, M. P. (2006). The relational self revealed: Integrative conceptualization of the implications and interpersonal life. Psychological Bulletin, 132(2), 151-179. http://dx.doi.org/10.1037/0033-2909.132.2.151

Côté, J. E., \& Levine, C. (1988). A critical examination of the ego identity status paradigm. Developmental Review, 8, 147-184. http://dx.doi.org/10.1016/0273-2297(88)90002-0

Côté, J., \& Levine, C. (2002). Identity formation, agency and culture: A social psychological synthesis. Mahwah, NJ: Lawrence Erlbaum Associates Inc.

Crocetti, E., Schwartz, S., Fermani, A., \& Meeus, W. (2010). The Utrecht Management of Identity Commitment Scale (U-MICS): Italian validation and cross-national comparisons. European Journal of Psychological Assessment, 26, 169-183. http://dx.doi.org/10.1027/1015-5759/a000024

Crocetti, E., Luyckx, K., Scrignaro, M., \& Sica, L. S. (2011). Identity formation in Italian emerging adults: A cluster-analytic approach and associations with psychosocial functioning. European Journal of Developmental Psychology, 8, 558-572. http://dx.doi.org/10.1080/17405629.2011.576858 
Crocetti, E., Scrignaro, M., Sica, L. S., \& Magrin, M. E. (2012). Correlates of identity configurations: Three studies with adolescent and emerging adult cohorts. Journal of Youth Adolescence, 41(6), 732-748. http://dx.doi.org/10.1007/s10964-011-9702-2

Epp, A. M., \& Price, L. L. (2008). Family identity: A framework of identity interplay in consumption practices. Journal of Consumer Research, 35(1), 50-57. http://dx.doi.org/10.1086/529535

Erikson, E. H. (1968). Identity: Youth and crisis. New York: Norton.

Eurostat (2016). Retirado de http://ec.europa.eu/eurostat/en/web/products-datasets/-/DEMO_NIND.

Evans, N. J., Forney, D. S., Guido, F. M., Patton, L. D., \& Renn, K. A. (2010). Student development in college (2 ${ }^{\mathrm{a}}$ Ed.). San Francisco: John Wiley, Sons, Inc.

Fornell, C. \& Larcker, D. F. (1981). Evaluating structural equation models with unobservable variables and measurement error. Journal of Marketing Research, 18(1), 39-50. http://dx.doi.org/10.2307/3151312

Garson, G. D. (2012). Structural Equation Modeling. Asheboro, NC: Statistical.

Guerreiro, M. D., \& Abrantes, P. (2004). Moving into adulthood in a Southern European country: Transitions in Portugal. Revista Portuguesa de Ciências Sociais, 3(3), 191-209.

Grotevant, H. D. (1987). Toward a process model of identity formation. Journal of Adolescent Research, 2, 203-222. http://dx.doi.org/10.1177/074355488723003

Hair, J. F., Black, W. C., Babin, B. J., \& Anderson, R. E. (2009). Multivariate data analysis (7rd Ed.). Upper Saddle River, NJ: Prentice Hall.

Iyer, A., Jetten, J., Tsivrikos, D., Postmes, T., \& Haslam, S. (2009). The more (and the more compatible) the merrier: Multiple group memberships and identity compatibility as predictors of adjustment after life transitions. British Journal of Social Psychology, 48(4), 707-733. http://dx.doi.org/10.1348/014466608X397628

Jablonski, J., \& Martino, S. D. (2013). A qualitative exploration of emerging adults' and parents' perspectives on communicating adulthood status. The Qualitative Report, 18(37), 1-12.

Kilstra, T. A., Hale, W. W., Raaijmakers, Q. A., Branje, S. J., \& Meeus, W. H. (2010). Identity formation in adolescence: Change or stability? Journal of Youth and Adolescence, 39(2), 150-162. http://dx.doi.org/10.1007/s10964-009-9401-4

Kroger, J. (2002). Introduction: Identity development through adulthood. Identity: An International Journal of Theory and Research, 2, 1-5. http://dx.doi.org/10.1207/S1532706XID0201_01

Kublikowsky, I., \& Rodrigues, C.M. (2016). 'Kangaroo generations': New contexts, new experiences. Estudos Psicológicos, 33(3), 535-542. http://dx.doi.org/10.1590/1982-02752016000300016

Linares, J. L. (1996). Identidad y narrativa - La terapia familiar en la práctica clínica. Barcelona: Paidós Terapia Familiar.

Luyckx, K., Goossens, L., Soenens, B., \& Beyers, W. (2006). Unpacking commitment and exploration: Preliminary validation of an integrative model of late adolescent identity formation. Journal of Adolescence, 29, 361-378. http://dx.doi.org/10.1016/j.adolescence.2005.03.008

Luyckx, K., Schwartz, S. J., Berzonsky, M. D., Soenens, B., Vansteenkiste, M., Smits, I., \& Goossens, L. (2008). Capturing ruminative exploration: Extending the four-dimensional model of identity formation in late adolescence. Journal of Research in Personality, 42, 58-82. http://dx.doi.org/10.1016/j.jrp.2007.04.004

Luyckx, K., Schwartz, S. J., Goossens, L., Beyers, W., \& Missotten, L. (2011). Processes of personal identity formation and evaluation. In S. J. Schwartz, K. Luyckx, \& V. L. Vignoles (Eds.), Handbook of identity theory and research (pp. 77-98). New York: Springer.

Luyckx, K., Klimstra, T., Duriez, B., Petegem, S., \& Beyers, W. (2013). Personal identity processes from adolescence through the late 20s: Age trends, functionality, and depressive symptoms. Social Development, 22, 707-721. http://dx.doi.org/10.1111/sode.12027

Luyckx, K., Klimstra, T., Schwartz, S., \& Duriez, B. (2013). Personal identity in college and the work context: Developmental trajectories and psychosocial functioning. European Journal of Personality, 27, 222-237. http://dx.doi.org/10.1002/per.1903

Mastrotheodoros, S., \& Motti-Stefanidi, F. (2016). Dimensions of Identity Development Scale (DIDS): A test of longitudinal measurement invariance in Greek adolescents. European Journal of Developmental Psychology, 14(5), 605-617. http://dx.doi.org/10.1080/17405629.2016.1241175

Marcia, J. E. (1966). Development and validation of ego-identity status. Journal of Personality and Social Psychology, 3, 551-558. http://dx.doi.org/10.1037/h0023281

Marôco, J. (2007). Análise estatística - Com utilização do SPSS (3ª Ed.). Lisboa: Edições Sílabo, Lda.

Marôco, J. (2010). Análise de equações estruturais - Fundamentos teóricos, software e aplicações. Pêro Pinheiro: ReportNumber, Lda. 
Meeus, W., Iedema, J., Helsen, M., \& Vollebergh, W. (1999). Patterns of adolescent identity development: Review of literature and longitudinal analysis. Developmental Review, 19, 419-461. http://dx.doi.org/10.1006/drev.1999.0483

Meeus, W., van de Schoot, R., Keijsers, L., Schwartz, S. J., \& Branje, S. (2010). On the progression and stability of adolescent identity formation: A five-wave longitudinal study in early-to-middle and middle-to-late adolescence. Child Development, 81, 1565-1581. http://dx.doi.org/10.1111/j.14678624.2010.01492.x.

Meeus, W. (2011). The study of adolescent identity formation 2000-2010: A review of longitudinal research. Journal of Research on Adolescence, 21, 75-94. http://dx.doi.org/10.1111/j.15327795.2010.00716.x

Mendonça, M., Andrade, C., \& Fontaine, A. (2009). Transição para a idade adulta e adultez emergente: Adaptação do Questionário de Marcadores da Adultez junto de jovens portugueses. Psychologica, $51,147-168$.

Mannerström, R., Hautamäki, A., \& Leikas, S. (2017). Identity status among young adults: Validation of the Dimensions of Identity Development Scale (DIDS) in a Finnish sample. Nordic Psychology, 69(3), 195-213. http://dx.doi.org/10.1080/19012276.2016.1245156

Morsunbul, U., \& Cok, F. (2014). The adaptation of the dimensions of identity development scale into Turkish. The Journal of Psychiatry and Neurological Sciences, 27, 6-14. http://dx.doi.org/10.5350/DAJPN2014270101

Pesigan, I. J. A., Luyckx, K., \& Alampay, L. P. (2014). Brief report: Identity processes in Filipino late adolescents and young adults: Parental influences and mental health outcomes. Journal of Adolescence, 37, 599-604. http://dx.doi.org/10.1016/j.adolescence.2014.04.012

Santos, P. J., Ferreira, J. A., \& Gonçalves, C. M. (2014). Indecisiveness and career indecision: A test of a theoretical model. Journal of Vocational Behavior, 85, 106-114. http://dx.doi.org/10.1016/j.jvb.2014.05.004.

Saraiva, L. M., \& Matos, P. M. (2015). Becoming an adult in Portugal: Negotiating pathways between opportunities and constraints. In R. Zukauskien, Emerging adulthood in a European context (pp. 117-137). New York: Routledge.

Schwartz, S. H., \& Rubel-Lifschitz T. (2009). Cross-national variation in the size of sex differences in values: Effects of gender equality. Journal of Personality and Social Psychology, 97(1), 171-185. http://dx.doi.org/10.1037/a0015546.

Schwartz, S. J., Zamboanga, B. L., Luyckx, K., Meca, A., \& Ritchie, R. (2013). Identity in emerging adulthood: Reviewing the field and looking forward. Emerging Adulthood, 1(2), 96-113. http://dx.doi.org/10.1177/2167696813479781

Sedikides, C., \& Brewer, M. B. (2001). Individual self, relational self, collective self. Psychology Press.

Sica, L. S., Sestito, L. A., \& Ragozini, G. (2014). Identity coping in the first years of university: Identity diffusion, adjustment and identity distress. Journal of Adult Development, 21, 159-172. http://dx.doi.org/10.1007/s10804-014-9188-8

Skhirtladze, N., Javakhishvili, N., Schwartz, S. J., Beyers, W., \& Luyckx, K. (2016). Identity processes and statuses in post-Soviet Georgia: Exploration processes operate differently. Journal of Adolescence, 47, 19-209. http://dx.doi.org/10.1016/j.adolescence.2015.08.006

Vieira, A. C. S., \& Rava, P. G. S. (2012). Ninho cheio: Perspectivas de pais e filhos. Psicologia: Teoria e Prática, 14(1), 84-96.

Vignoles, V. L., Schwartz, S. J., \& Luyckx, K. (2011). Introduction: Toward an integrative view of identity. In S. J. Schwartz, K. Luyckx, \& V. L. Vignoles (Eds.), Handbook of identity theory and research (pp. 127). New York: Springer.

Waterman, A. S., \& Archer, S. L. (1990). A life-span perspective on identity formation: Development in form, function, and process. In P. B. Baltes, D. L. Featherman, \& R.M. Lerner (Eds.), Life-span development and behavior (vol. 10, pp. 30-57). Hillsdale, NJ: Erlbaum.

Zimmermann, G., Lannegrand-Willems, L., Safont-Mottay, C., \& Cannard, C. (2015). Testing new identity models and processes in French-speaking adolescents and emerging adults students. Journal of Youth and Adolescence, 44, 127-141. http://dx.doi.org/10.1007/s10964- 013-0005-7

Historial do artigo

Recebido 06/11/2016

Aceite $\quad 11 / 06 / 2018$

Publicado 12/2018 